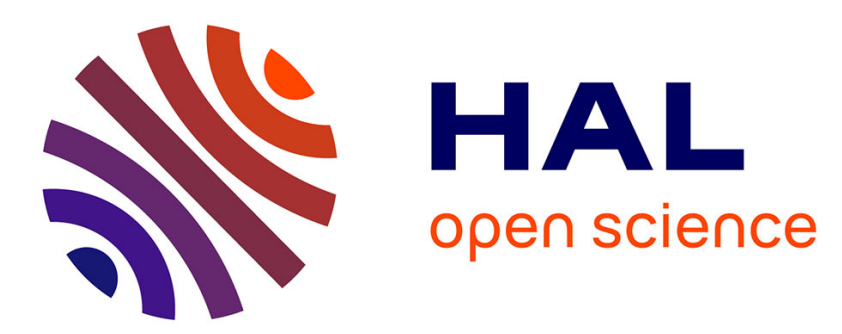

\title{
Sonification of Golf Putting Gesture Reduces Swing Movement Variability in Novices
}

Benjamin O'Brien, Brett Juhas, Marta Bieńkiewicz, Frank Buloup, Lionel Bringoux, Christophe Bourdin

\section{- To cite this version:}

Benjamin O'Brien, Brett Juhas, Marta Bieńkiewicz, Frank Buloup, Lionel Bringoux, et al.. Sonification of Golf Putting Gesture Reduces Swing Movement Variability in Novices. Research Quarterly for Exercise and Sport, 2020, pp.1-10. 10.1080/02701367.2020.1726859 . hal-02495502

\section{HAL Id: hal-02495502 \\ https://hal-amu.archives-ouvertes.fr/hal-02495502}

Submitted on 19 Apr 2021

HAL is a multi-disciplinary open access archive for the deposit and dissemination of scientific research documents, whether they are published or not. The documents may come from teaching and research institutions in France or abroad, or from public or private research centers.
L'archive ouverte pluridisciplinaire HAL, est destinée au dépôt et à la diffusion de documents scientifiques de niveau recherche, publiés ou non, émanant des établissements d'enseignement et de recherche français ou étrangers, des laboratoires publics ou privés. 


\section{Sonification of golf putting gesture reduces swing movement 2 variability in novices}

This study investigates whether novices can use sonification to enhance golf putting performance and swing movements. Forty participants first performed a series of $2 \mathrm{~m}$ and $4 \mathrm{~m}$ putts, where swing velocities associated with successful trials were used to calculate their mean velocity profile (MVP). Participants were then divided into four groups with different auditory conditions: static pink noise unrelated to movement, auditory guidance based on personalized MVP, and two sonification strategies that mapped the real-time error between observed and MVP swings to modulate either the stereo display or roughness of the auditory guidance signal. Participants then performed a series of $2 \mathrm{~m}$ and $4 \mathrm{~m}$ putts with the auditory condition designated to their group. In general our results showed significant correlations between swing movement variability and putting performance for all sonification groups. More specifically, in comparison to the group exposed to static pink noise, participants who were presented auditory guidance significantly reduced the deviation from their average swing movement. In addition, participants exposed to error-based sonification with stereo display modulation significantly lowered their variability in timing swing movements. These results provide further evidence of the benefits of sonification for novices performing complex motor skill tasks. More importantly, our findings suggest participants were able to better use online error-based sonification rather than auditory guidance to reduce variability in the execution and timing of their movements.

Keywords: auditory guidance; error-based sonification; motor control; golf

\section{Introduction}

Complex motor skill performance improvement can pertain to a myriad of things, from goal attainment to movement efficiency and consistency. Humans of course are multisensory, but vision is regarded as the primary sensory modality for provision of feedback in the performance of complex motor tasks and goal attainment (Zhao \& Warren, 2014). However, findings from recent studies suggest other senses play important roles in the guiding of motor actions (Arnott \& Alain, 2011; Kohler et al., 
2002; Sigrist, Rauter, Riener, \& Wolf, 2013). In this study we examined whether novices can use sonification, the mapping of data onto sound, to enhance golf putting performance and swing movement.

Real-time (“online”) sonification has been proven to enhance the performance of motor control tasks (Schaffert et al., 2019; Sigrist et al., 2013). Thoret et al. (2014) found participants enhanced their ability to perceive and associate movement profiles when presented acoustic information concurrent with their movements. Dyer, Rodger, \& Stapleton (2016) found that, by repeating motor tasks with synchronous sound, participants recreated these actions more easily. Similar benefits of online artificial sonification have been shown in sports training studies, such as rowing (Effenberg, Ursula, Schmitz, Krueger, \& Mechling, 2016; Dubus \& Bresin, 2014) and cycling (Sigrist, Fox, Riener, \& Wolf, 2016). performance. In this way, sonification functions like an index that points to an error or deviation from an ideal motor action. van Vugt \& Tillmann (2015) found that participants engaged with error-based sonification improved motor regularity when performing tapping tasks. Dailly et al. (2012) similarly reported that participants who were presented error-based sonification significantly reduced their spatial error completing a simple figure-tracing task. Wolf et al. (2011) showed that novice

51 participants were able to immediately use auditory feedback to enhance their rowing 52 performance by reducing spatial and temporal errors during training. However, none of

53 the aforementioned studies focused on the effects of error-based sonification on complex motor tasks.

An example of a complex motor skill is golf putting (Wulf \& Shea, 2002; Frank et al., 2013), a gesture with well defined sub-movements and, due to the design of the 
putter club, requires a clear translation from the person's movement velocity to energy, so the ball can travel the distance required. It also requires visual concentration on the ball before making contact. Because of this, there is an opportunity to stress other sensory cues for motor-skill guidance. Keogh \& Hume (2012) demonstrated that a primary focus in golf training is kinematics and posited that errorless learning might be afforded by using different visual feedback strategies. A similar approach that replaces visual with auditory feedback may prove to be particularly useful, as it would free attentional resources required to visually monitor club and ball positions. Interestingly, only a handful of comprehensive studies focus on the effects of sonification in golf training. Kleiman-Weiner \& Berger (2006) developed a method that mapped, among other things, the club head velocity of an expert golfer performing the golf swing to different sound parameters, such as pitch and vowel synthesis formants, but no findings were reported. Bieńkiewicz et al. (2019) investigated motor learning of putting tasks in novices when presented either visual or auditory information developed

71 from the swing velocity of an expert golfer. In comparison to the control group, who were not presented any additional sensory information, novices had lower variability of their movements (measured as the standard deviation of impact velocity across trials) and were putting closer to the target when presented either visual or auditory sensory information. In addition a pilot study reported by O’Brien et al. (2018) found novices were able to identify swing speed as represented by auditory signals. Similarly, Murgia et al. (2017) found golfers were able to recognise their own idiosyncratic swings via sonification, which demonstrates the relationship between performing golf swings and perceiving sounds based on them. A distinguishing feature of this study was to focus on the effects of error-based sonification on putting performance in novices. 
82 (2018) showed a significant correlation between left forearm segment variability and

83 horizontal launch angle and suggested that by reducing their variability, golfers might

84 enhance their performance. The authors also proposed that golfers employ different

85 putting styles, which vary between more stable and flexible motor outputs. As they

86 concluded, additional research into movement variability and putting is needed to

87 confirm this proposition, which asserts some practical implications, as golf instructors

88 might prioritize identifying whether a golf pupil utilizes movement variability or has a

89 more consistent swing profile. Thus, we wanted to look more deeply into the

90 relationship between performance variability and goal attainment. Expanding on this,

91 we wanted to examine whether sonification could help reduce complex motor

92 performance variability, which in turn might affect putting performance.

93 It was important to select an important feature in golf putting for which to

94 measure, model, and use to compare and calculate performance errors in real-time. A

95 fundamental factor in the success of a golf putt is swing speed (Burchfield \&

96 Venkatesan, 2010), which was further evidenced by Craig, Delay, Grealy, \& Lee (2000)

97 who reported club head velocity at impact strongly correlates to ball distance. However

98 the golf putting gesture is also uniquely personal, as there are many ways to swing the

99 putter club, such as increasing or decreasing wrist movement.

100 Our first objective then was to develop a method of sonification that was

101 participant-dependent, so as to accurately reflect swing idiosyncrasies and, moreover,

102 personalize the sounds presented to participants. We decided to present participants

103 auditory guidance based on their individual average swing performance, which was

104 calculated following a series of successful putts at different distances. A major 
advantage of this method is that it adjusts to the kinematic capacities of the individual, which may prove useful in both healthy and rehabilitation research. performance and swing movements by using online sonification based on errors of

109 performance. Our second goal was to develop an online sonification method that maps

110 performance errors in ways that modulated the auditory guidance signal. Although it is

111 known that healthy humans do not perceive sound similarly due to their physiological

112 and psychological differences, a study by Johnson, Watson, \& Jensen (1987) found

113 patterns identified in healthy participants affected auditory performance similarly.

114 Based on these findings, we decided to develop different methods for modulating the

115 auditory guidance signal in real-time, so as to maximise the opportunity for participants

116 to perceive and use sonification based on errors of performance.

\section{Methods}

118 Participants

119 Forty right-handed participants (28 male; mean age: 22.4; standard deviation: 7.2)

120 affiliated with ----- participated in the experiment. All participants self-reported good or

121 corrected vision and normal hearing. All participants consented to voluntary

122 participation in the study and were informed of their right to withdraw at any time. This

123 study was performed in accordance with the ethical standards of the Declaration of

124 Helsinki (Salako, 2006). The Ethics Committee of ----- approved the protocol.

\section{Experimental setup}

126 Participants used an Odyssey White Ice putter (length: $0.97 \mathrm{~m}$; weight: $0.59 \mathrm{~kg}$ ) to hit

127 Titleist PRO V1X balls. A synthetic grass terrain was used (length: 5 m; width: $1.8 \mathrm{~m}$ ).

128 White circles with $0.11 \mathrm{~m}$ diameters were painted at the starting position and the $2 \mathrm{~m}$ 
129 and $4 \mathrm{~m}$ target distances. Participants wore Sennheiser headphones when presented

130 sound.

131 The Codamotion CX1 Scanner was used to collect club kinetic data (sampling

132 rate: $200 \mathrm{~Hz}$ ). The CX1 Scanner was placed $2 \mathrm{~m}$ away from participants with $1 \mathrm{~m}$

133 elevation. Two infra-red active markers were placed near the club head at the bottom of

134 the club shaft and just below the handgrip.

\section{Procedure}

136 Participants first completed 20 Baseline trials at $2 \mathrm{~m}$ and $4 \mathrm{~m}$ (total: 40 trials). Unless

$13720 \%^{1}$ of their putts at both distances were within $0.25 \mathrm{~m}$ of the target, they were

138 excluded from the study. Participants were then randomly assigned to one of four

139 experimental groups $(n=10)$. Following a pause required to calculate their mean

140 velocity profile (MVP) (see: Protocol), participants completed two rounds of 20

141 Experimental trials at $2 \mathrm{~m}$ and $4 \mathrm{~m}$ (total: 80 trials, counterbalanced). Each participant

142 performed 120 putts in total over the course of the experiment. Participants were only

143 presented sound during Experimental trials.

\section{Protocol}

145 A custom program developed in Python streamed and recorded all values monitored by

146 CodaMotion. To present personalized MVPs to participants, their successful Baseline

147 trials were selected and synchronised at impact point, where after their club head

148 velocities were shifted and averaged offline. During the Experimental trials we

149 estimated the time to impact with the ball by using club head marker values to calculate

\footnotetext{
${ }^{1}$ We decided that $20 \%$ was the minimum number of trials required to provide participants with auditory guidance or error-based sonification that faithfully represented their swing idiosyncrasies.
} 
150 its velocity and distance from the ball. Once the backswing velocity reached a minimum

151 threshold of $0.1 \mathrm{~m} . \mathrm{s}$, we began the process of comparing the current position of the club

152 head with the starting position of the club (near the ball) and the current club head

153 velocity with the MVP. Error was then calculated by comparing the current estimated

154 time to impact with the MVP time of impact. This estimated time to impact was then

155 compared in real-time to the participant's MVP, which, in turn, gave us a real-time

156 difference, or error, between her observed and MVP swings. Figure 1 illustrates the

157 real-time error between a participant's observed and MVP swings for a $2 \mathrm{~m}$ putt.

158 Figure 1 Top: comparison between observed (blue) and MVP (black) swings. Bottom: 159 error (red)
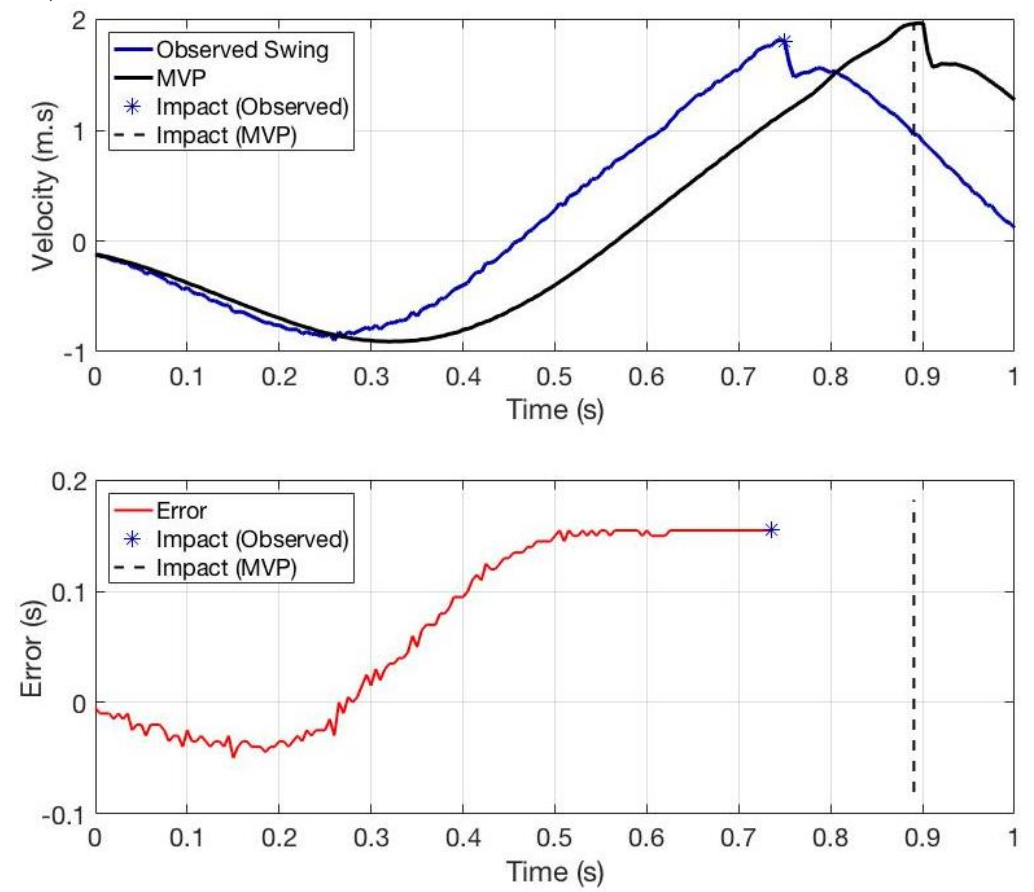

161 Before each trial, participants were asked to place the club head close to the ball and

162 remain motionless for approximately $1 \mathrm{~s}$. This allowed us to accurately monitor a

163 significant change in velocity - the start of the backswing. Once identified, velocity and

164 error information was transmitted locally to a computer running Max/MSP, which was

165 used for sound synthesis. Sound was presented to participants at the start of their

166 backswing during the Experimental trials. 
168 Each group was presented a different auditory condition. 'Control' group participants

169 were presented static pink noise that was independent of observed movements and was

170 the same across all Experimental trials. The duration of the static pink noise was equal

171 to that of their MVP. 'MVP' group participants were presented auditory guidance based

172 on their personalized MVPs, where velocity values were sequenced and mapped to the

173 frequency of a sinusoidal oscillator. As described in O'Brien et al. (2018), this strategy

174 was based on discussions with golf instructors and trainers, who frequently whistled

175 upwards and then downwards to describe, in general, putting mechanics. The absolute

176 values of velocities were linearly mapped and scaled to a frequency range of $80-2000$

$177 \mathrm{~Hz}$ and transformed to a Mel scale (122 - 1521 mels). This sound was the same across

178 the Experimental trials (for each distance) and was independent of observed

179 movements. Because the sounds presented to both Control and MVP participants were

180 independent of observed movements, they were considered "offline."

181 The remaining two groups were presented online sonification based on the

182 calculated errors between observed and MVP swings. Similar to the MVP group, both

183 groups were presented auditory signals generated by mapping and scaling velocity

184 values to the frequency of a sinusoidal oscillator, however they were modulated

185 differently depending on the group. In both cases, the magnitude of the error was

186 directly mapped to the magnitude of the modulation. The 'Directivity' group was

187 presented online sonification based on stereo display, where the auditory signal was

188 panned right if the error was negative (and vice-versa). This design was based on a

189 study by Libkum, Otani, \& Steger (2002), which found participants who trained by

190 synchronising their hands and feet with a stereophonic metronome improved

191 performance. The 'Roughness' group was presented online sonification based on error 
192 sign to modulate the roughness ${ }^{2}$ of the auditory signal: if negative, it was processed by a

193 Coulomb friction sound synthesiser to become more "grating" if positive, it was

194 modulated by a von Kármán model (Diedrich \& Drischler, 1957) to evoke wind speeds.

195 The Supplementary Materials demonstrate the differences between all auditory

196 conditions.

\section{Data processing and statistics}

198 To investigate whether sonification affected putting performance, we examined the

199 distance between the final location of the ball and the target - the target distance error.

200 Both target distance error mean $\left(T D E_{\mu}\right)$ and standard deviation $\left(T D E_{\sigma}\right)$ were used in our

201 analysis of all Baseline and Experimental trials. In addition, we calculated the

202 percentage of improvement for both $T D E_{\mu}$ and $T D E_{\sigma}$ by dividing the difference

203 between Baseline and Experimental trials by Baselines trials and multiplying it by 100 .

204 To investigate the effects of sonification on movement and timing variability,

205 we examined participant deviation from average swing speed and temporal ratio,

206 respectively. To measure the former, we synchronised trials at impact, shifted their

207 velocities to the time of impact, and then calculated the Normalised Root Mean

208 Standard Deviation from their MVP (1), where $\hat{x}$ represents participant MVP, $x$ is the

209 collection of velocity values from the start of the backswing up to impact for trial $n$, and

$210 N$ is the number of successful trials. These deviations were then averaged $\left(N R M S D_{\mu}\right)$.

211 To measure temporal ratio variability $\left(T R_{\sigma}\right)$, we calculated the standard deviation of the

212 temporal ratio, which is the ratio of the backswing duration to downswing duration.

213 Because sonification was developed from participant MVPs, which were based on the

\footnotetext{
${ }^{2}$ A multimodal descriptor of texture, roughness can be simulated in the auditory domain by using a number of methods, including amplitude modulation (Zwicker \& Fastl, 1999) and physical modelling (Conan et al., 2014).
} 
214 swing profiles associated with successful trials, we excluded all Baseline and

215 Experimental trials with putts that were greater than $0.25 \mathrm{~m}$ from the target from our

216 analysis of swing movement and timing. In addition, we calculated a percentage of

217 improvement for swing movement and timing variability based only on successful

218 trials.

$$
N R M S D=\frac{\sqrt{\frac{\sum_{n=1}^{N}\left(\hat{x}-x_{n}\right)^{2}}{N}}}{x_{\max }-x_{\min }}
$$

220 For all outcome variables, mixed ANOVAs were carried out with group as a between-

221 subjects factor and both target distance and trial type (Baseline, Experimental) as within-subject factors. Where main effects were detected, post-hoc Bonferroni-adjusted t-tests were carried out. All significant post-hoc findings were reported $(X \pm Y)$ with $X$ mean difference and $Y$ standard error. Where the assumption of sphericity was violated,

225 Greenhouse-Geisser adjustments are reported.

\section{Preliminary analysis}

227 All participants were included in our analysis. At first glance it appeared participants

228 found the $2 \mathrm{~m}$ target (mean target distance error: $0.44 \mathrm{~m}$; SD target distance error: 0.14 $\mathrm{m}$ ) to be less difficult than the $4 \mathrm{~m}$ target (mean target distance error: $0.62 \mathrm{~m}$; SD target distance error: $0.16 \mathrm{~m})$. Repeated measures ANOVA tests revealed main effects on mean target distance error $\mathrm{F}_{1,3}=47.51, p<0.001, \eta_{p}^{2}=0.94$ and SD target distance error $\mathrm{F}_{1,3}=15.53, p<0.001, \eta_{p}^{2}=0.67$. Our preliminary observations were substantiated by post-hoc tests that revealed mean target distance error at $2 \mathrm{~m}$ was

234 significantly less than $4 \mathrm{~m}(0.18 \pm 0.03), p<0.001$. Similarly participants showed 235 significantly lower SD target distance error at $2 \mathrm{~m}$ when compared to $4 \mathrm{~m}(0.12 \pm 0.03)$, 236 $p<0.001$ 


\section{Results}

\section{Target Distance Error}

239 We first examined the percentage of improvement for mean target distance error

$240\left(T D E_{\mu}\right)$ at $2 \mathrm{~m}$ and $4 \mathrm{~m}$ and found a main effect on distance $\mathrm{F}_{1,3}=5.11, p<0.05, \eta_{p}^{2}=$

2410.38 , but no group effects, $p>0.05$. Post-hoc tests revealed participants significantly

242 improved their percentage of improvement for $T D E_{\mu}$ at $2 \mathrm{~m}$ when compared to $4 \mathrm{~m}$

$243(9.38 \pm 4.15), p<0.05$.

Next, to examine the effects of sonification on putting performance, we compared $T D E_{\mu}$ during Baseline and Experimental trials at $2 \mathrm{~m}$ and $4 \mathrm{~m}$ and found main effects on distance $\mathrm{F}_{1,3}=108.47, p<0.001, \eta_{p}^{2}=0.94$ and trial type $\mathrm{F}_{1,3}=37.61, p$

$<0.001, \eta_{p}^{2}=0.93$, but no significance on group, $p>0.05$. Post-hoc tests showed participants were closer to the target at $2 \mathrm{~m}(18.77 \pm 1.8)$ and during the Experimental trials $(10.18 \pm 1.66), p<0.001$

Similarly, we first examined the percentage of improvement for standard deviation of target distance error $\left(T D E_{\sigma}\right)$ at $2 \mathrm{~m}$ and $4 \mathrm{~m}$ and found no significance for neither group nor distance, $p>0.05$.

Next we compared $T D E_{\sigma}$ during Baseline and Experimental trials at $2 \mathrm{~m}$ and 4 $\mathrm{m}$ and similarly found main effects on distance $\mathrm{F}_{1,3}=43.9, p<0.001, \eta_{p}^{2}=0.82$ and trial type $\mathrm{F}_{1,3}=31.56, p<0.001, \eta_{p}^{2}=0.85$ and a distance $*$ group interaction $\mathrm{F}_{3,36}=$ 3.13, $p<0.05, \eta_{p}^{2}=0.21$. Post-hoc tests showed participants performed with lower variability at $2 \mathrm{~m}(12.22 \pm 1.8)$ and during the Experimental trials $(8.74 \pm 1.56), p<$ 0.001. Additionally, the following groups had significantly lower variability at $2 \mathrm{~m}$ rather than at $4 \mathrm{~m}, p<0.001$ : Control $(13.37 \pm 3.69)$, Directivity $(11.27 \pm 3.69)$, and

260 Roughness $(20.04 \pm 3.69)$. 
262 We examined the percentage of improvement for average swing velocity deviation from $\operatorname{MVP}\left(N R M S D_{\mu}\right)$ trials at $2 \mathrm{~m}$ and $4 \mathrm{~m}$ and found main effects on group $\mathrm{F}_{3,36}=3.17, p<$ $2640.05, \eta_{p}^{2}=0.21$ and distance $\mathrm{F}_{1,3}=6.62, p<0.01, \eta_{p}^{2}=0.67$. Post-hoc tests revealed the 265 MVP group significantly improved in comparison to the Control group (25.2 \pm 8.56$), p$ $<0.05$ (Figure 2). There were no other significant differences between groups, $p>$

267 0.05. When compared to the $4 \mathrm{~m}$ target, participants improved performance at $2 \mathrm{~m}$

$268 \quad(18.27 \pm 6.52), p<0.05$.

269 Figure 2 Percentage of improvement for average swing velocity deviation from MVP of 270 successful trials at $2 \mathrm{~m}, 4 \mathrm{~m}$ by group.

271

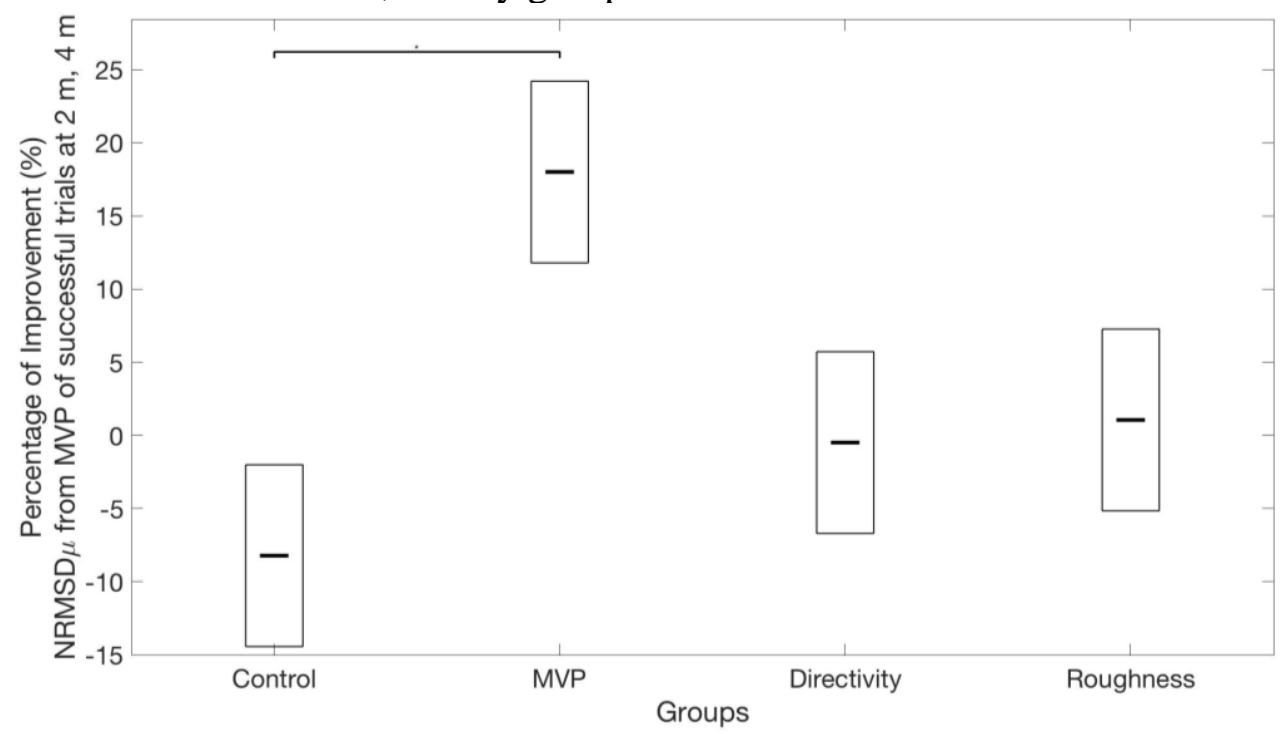

272 Next we examined participant $N R M S D_{\mu}$ from during Baseline and Experimental trials

273 at $2 \mathrm{~m}$ and $4 \mathrm{~m}$, where we observed main effects on distance $\mathrm{F}_{1,3}=14.63, p<0.001, \eta_{p}^{2}$

$274=0.8$, trial type $\mathrm{F}_{1,3}=14.93, p<0.001, \eta_{p}^{2}=0.57$, and interactions on trial type $*$ group

$275 \mathrm{~F}_{3,36}=3.76, p<0.05, \eta_{p}^{2}=0.24$. Post-hoc tests revealed participants significantly

276 lowered their $N R M S D_{\mu}$ at $4 \mathrm{~m}(0.64 \pm 0.17)$ and during Experimental trials $(0.61 \pm$

277 0.16), $p<0.001$. Additionally, participants in the MVP group significantly lowered

278 their $N R M S D_{\mu}$ during Experimental trials $(1.5 \pm 0.31), p<0.001$ (Figure 3). 
Figure 3 Average swing velocity deviation from MVP of successful baseline and experimental trials at $2 \mathrm{~m}, 4 \mathrm{~m}$.

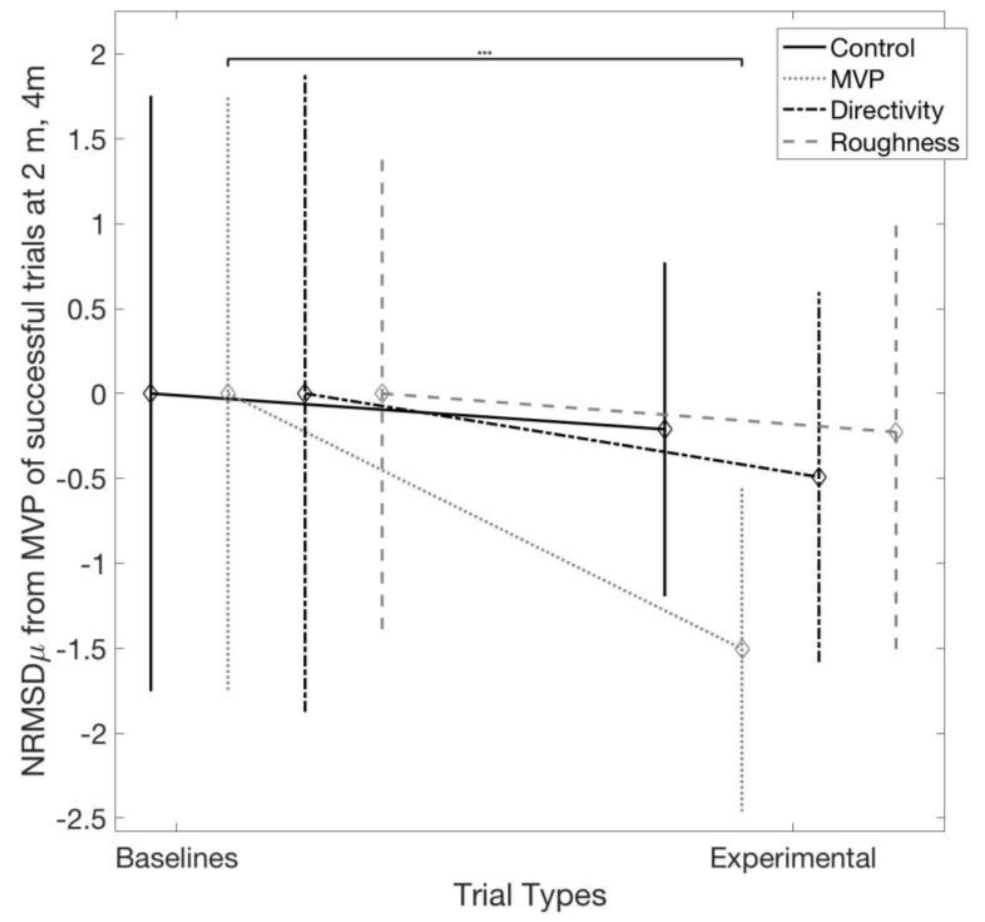

\section{Temporal ratio}

283 We first examined the percentage of improvement for standard deviation of temporal ratio $\left(T R_{\sigma}\right)$ trials at $2 \mathrm{~m}$ and $4 \mathrm{~m}$ and found no significance for neither group nor distance, $p>0.05$.

Next we examined participant $T R_{\sigma}$ during Baseline and Experimental trials at 2 $\mathrm{m}$ and $4 \mathrm{~m}$, and we observed main effects on trial type $\mathrm{F}_{1,3}=7.68, p<0.01, \eta_{p}^{2}=0.46$ and interactions on trial type $*$ group $\mathrm{F}_{3,36}=3.02, p<0.05, \eta_{p}^{2}=0.2$, distance $*$ group

$289 \mathrm{~F}_{3,36}=3.28, p<0.05, \eta_{p}^{2}=0.21$, and distance $*$ trial type $*$ group $\mathrm{F}_{3,36}=3.22, p<0.05$ $290 \quad \eta_{p}^{2}=0.21$. Post-hoc tests revealed participants significantly lowered their $T R_{\sigma}$ during

291 Experimental trials $(0.05 \pm 0.02), p<0.01$. The Directivity group significantly lowered 292 their $T R_{\sigma}$ during Experimental trials $(0.13 \pm 0.04), p<0.01$, when compared to

293 Baseline trials (Figure 4); during $2 \mathrm{~m}$ trials $(0.07 \pm 0.03), p<0.05$, when compared to

$2944 \mathrm{~m}$ trials; and during Experimental trials at $4 \mathrm{~m}(0.2 \pm 0.06), p<0.01$, when compared 
to Experimental trials at $2 \mathrm{~m}$. The Control group significantly lowered their $T R_{\sigma}$ during

$4 \mathrm{~m}(0.07 \pm 0.03), p<0.05$, when compared to $2 \mathrm{~m}$ trials, and Experimental trials at 2

$297 \mathrm{~m}(0.14 \pm 0.04), p<0.05$, when compared to Experimental trials at $4 \mathrm{~m}$.

298 Figure 4 Temporal ratio standard deviation of successful baseline and experimental 299 trials at $2 \mathrm{~m}, 4 \mathrm{~m}$ by group.

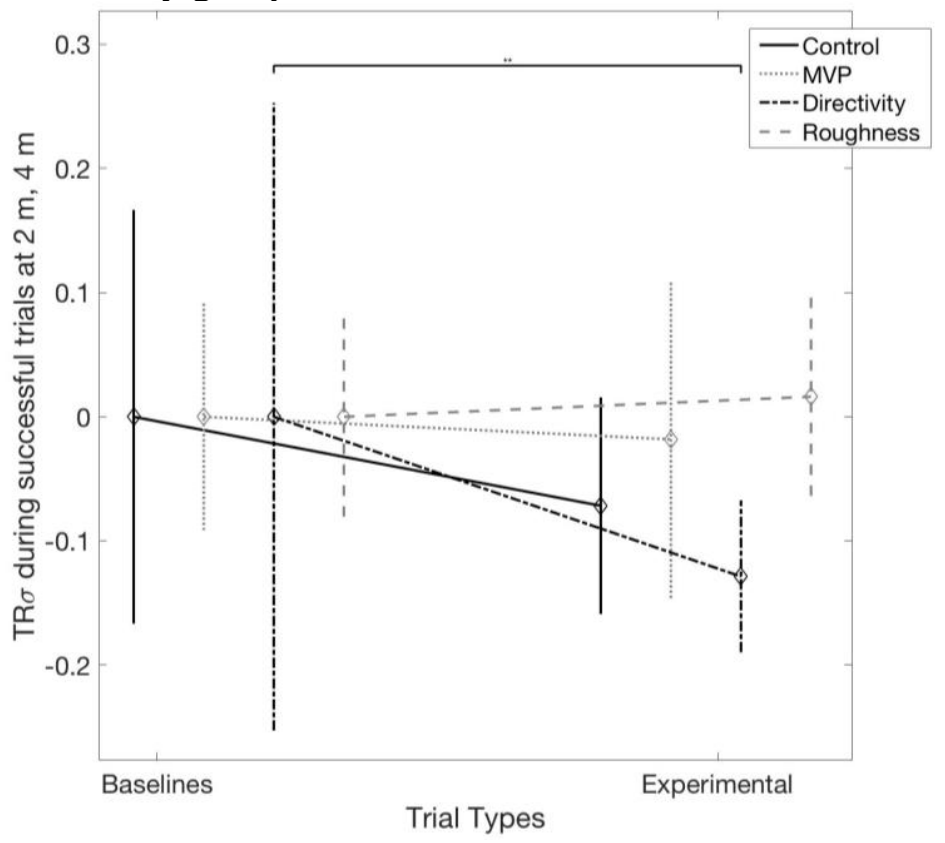

Correlations between putting performance and swing movement variability

302 Noting our significant findings for average swing velocity deviation from MVP for the MVP group and temporal ratio standard deviation for the Directivity group, we wanted

304 to test if any of the groups had significant correlations between putting performance

305 (target distance error mean and standard deviation) and swing movement variability

306 (deviation from average swing velocity, temporal ratio standard deviation). Using linear

307 regression models, Table 1 illustrates the Group $\mathrm{R}^{2}$ coefficients and $p$-values for

308 relationships between putting performance and swing movement variability, where:

$309 T D E_{\mu}$ and $T D E_{\sigma}$ are the target distance error mean and standard deviation, respectively;

$310 N R M S D_{\mu}$ is the average swing velocity deviation from MVP; and $T R_{\sigma}$ is the temporal

311 ratio standard deviation. 
312 Table 1 Group $\mathrm{R}^{2}$ coefficients and $p$-values for correlations between putting

313 performance and swing movement variability variables

\begin{tabular}{|c|c|c|c|c|c|c|c|c|}
\cline { 2 - 9 } \multicolumn{1}{c|}{} & \multicolumn{4}{c|}{ TDE $\mu$} & \multicolumn{3}{c|}{ TDE $\sigma$} \\
\cline { 2 - 9 } \multicolumn{1}{c|}{} & \multicolumn{2}{c|}{ NRMSD $\mu$} & \multicolumn{2}{c|}{ TR $\sigma$} & \multicolumn{2}{c|}{ NRMSD $\mu$} & \multicolumn{2}{c|}{ TR $\sigma$} \\
\hline Group & $R^{2}$ & $p$ & $R^{2}$ & $p$ & $R^{2}$ & $p$ & $R^{2}$ & $p$ \\
\hline Control & 0.07 & & 0.08 & & 0.09 & & 0.08 & \\
\hline MVP & 0.27 & & 0.54 & $*$ & 0.1 & & 0.57 & $*$ \\
\hline Directivity & 0.67 & $*$ & 0.69 & $* *$ & 0.82 & $* * *$ & 0.63 & $* *$ \\
\hline Roughness & 0.72 & $* *$ & 0.21 & & 0.63 & $* *$ & 0.16 & \\
\hline
\end{tabular}

314

315

316

317

318

319

320

321

322

323

where $\{*, * *, * * *\}$ mark significance for $p<\{0.05,0.01,0.001\}$

As expected, there were no significant correlations between putting performance and

swing movement variability for the Control group, while the MVP and Roughness

groups both reported strong correlations with putting performance, but only with

temporal ratio standard deviation and average swing velocity deviation, respectively.

Notably, only the Directivity group had significant correlations for all putting

performance-swing movement variability combinations.

\section{Discussion}

\section{Putting performance}

326 The goal of our study was to investigate whether novices were able to use sonification

327 to improve golf putting performance and reduce swing movement variability. While

328 participants significantly improved their target distance error average by $0.10 \pm 0.02 \mathrm{~m}$

329 and standard deviation by $0.09 \pm 0.02 \mathrm{~m}$ during the Experimental trials, we reported no

330 group effects. In addition, though the percentage of improvement was positive for mean 
target distance error, there were no group differences in the magnitude of the percentage

332 improvement. Because participants exposed to static pink noise similarly improved to

333 those who were presented auditory guidance or error-based sonification, at first glance

334 these results suggest performance enhancement was not influenced by the presence of

335 artificial sound, but rather based on movement familiarisation. There are, of course,

336 countless factors that contribute to golf putting performance, which have been the

337 subject of study, such as the putting green (Pataky \& Lamb, 2018). This point is

338 underlined by a report by Kammerer, Menshik, Erlemann, \& Lafortune (2014), which

339 found putting robots made only $80 \%$ putts at $5 \mathrm{~m}$. These observations taken together

340 suggest that when studying its effect on novices, sonification may play a more

341 important role enhancing putting movements, rather than directly influencing ball

342 distance from the target.

\section{$343 \quad$ Swing movement variability}

344 Our analysis showed swing movement variability was enhanced differently among groups. The MVP group showed a $25.2 \pm 8.56 \%$ greater percentage of improvement for deviation from average swing velocity when compared to the Control group. This important finding demonstrates the benefits of personalized sonification, which, in this case, was based on the average speed of successfully executed golf putts. Similar

349 benefits were reported in a study by Bieńkiewicz et al. (2019), which found novices improved putting performance when presented sonification based on the club head velocity of an expert golfer performing putts at multiple distances. However, unlike their study, where participants trained with sonification over an eight-week period, the MVP group enhanced its performance when presented personalized sonification, as it

354 improved its average swing movement variability. This point is underscored by our results that found MVP participants significantly reduced their deviation from average 
swing velocity $\left(N R M S D_{\mu}\right)$ during Experimental trials by $1.5 \pm 0.31$ residuals. An important distinction then between the two studies is that, while their study focused on examining the effects of sonification on learning the golf putting gesture, we examined and found participants were able to use auditory guidance based on their unique physiological constraints to enhance their movement by reducing variability. auditory guidance presented to MVP participants, although personalized, was independent of their swing movements. Thus despite also being fixed and unchanged by movement, participants were able to enhance their performance, reducing deviations from their average swing velocity during putts. These results support similarly reported findings regarding the benefits of repeated trainings with auditory information (Agostini et al, 2004; Young, Rodger, \& Craig, 2014). Our results suggest that, through repetition, the auditory guidance presented to the MVP participants allowed them to more clearly perceive the transition between the backswing and downswing, which, in turn allowed them to reduce their deviation from average swing velocity. Specifically, at the start of the downswing, velocity is zero, and, due to our method of mapping velocity to frequency, no sound was produced. This absence of sound or silence may have functioned like an index for users, which allowed them to assess their movements: if they finished their backswing before or after the silence, then they were to fast or slow, respectively. This idea of studying the effects of removing sound during the execution of complex movements is certainly interesting and appears to have not been extensively studied.

Although both Directivity and Roughness groups were presented online sonification based on errors of performance by modifying the same type of auditory 
381 sound to significantly reduce variability in the timing of their swing movements.. As the

382 timbre between the sounds presented to both MVP and Directivity groups was the same,

383 the major difference was the latter presented online sonification based on performance.

384 By modifying the stereo display of the auditory guidance signal, Directivity participants

385 were given additional information for which to perceive, interpret, and then use to

386 reduce the variability in the timing of their swing movements. Our findings support

387 those reported by Libkum, Otani, \& Steger (2002), who found training with auditory

388 stimuli improved putting performance, and add evidence to the role of sound

389 spatialization on human movement (Gandemer et al., 2017).

390 These findings also stress the importance of the sonification strategy and use of

391 simpler sounds. As Roughness group participants were also presented online

392 sonification based on errors of performance, the constantly shifting timbres may have

393 been too difficult for them to use. If we compare our average swing deviation and

394 temporal ratio standard deviation results for the Directivity and Roughness groups, our

395 findings suggest error-based sonification might be easier to use if either a combination

396 of simpler sounds - less complex - or two-dimensional displays are presented.

397 Nevertheless, the observed differences between groups illustrate the importance of

398 considering the inter-individual differences in which humans perceive sound - artificial

399 or otherwise - and possibly use information encoded in it while performing new and

400 complex motor tasks. A study by Wu et al. (2014) demonstrated a relationship between

401 the variability in successive movements and motor learning in novice participants. By

402 exploring different movement parameters, humans are able to refine newly acquired

403 actions and assess their movements and limitations, and our results suggest sound can

404 be an important actor in highlighting these differences. 


\section{What does this article add?}

406 In general, the results of our study provide further evidence of the benefits of

407 sonification for novices performing new complex motor skills. Our findings suggest

408 personalized templates for sonification help reduce variability in the execution and

409 timing of complex motor tasks. In addition, the significant correlations between putting

410 performance and swing movement variability reported for groups who were presented

411 online sonification based on performance errors add further support to the theory that

412 concurrent sonification can enhance feedback while performing motor-related tasks

413 (Dyer, Stapleton, \& Rodger, 2017). With follow up research, may be used to estimate

414 performance. Our results emphasise the potential impact of conveying temporally

415 accurate information based on errors of performance to novices performing new motor-

416 related tasks. These observations lend themselves to new questions regarding whether

417 errors are essential for complex motor task development and when does stabilizing

418 variability become beneficial.

419 Although we reported that sonification produced effects on swing movement

420 and timing variability, it did not affect the overall accuracy of the shot. This finding

421 suggests that participants were able to extract information regarding deviations from

422 their average swing performance from the synthesized sound, but it did not aid the

423 accuracy of their shots in comparisons to other groups. It is important to note that motor

424 variability plays an important role in motor learning processes and allows one to explore

425 the links between different spatiotemporal dynamics of movement and the outcome of

426 action (Bonassi et al., 2017). By providing error-based real time feedback we might

427 have hindered the natural unfolding of these processes by directing the attention of

428 participants to keeping the movement as consistent as possible. Unfortunately, we did

429 not introduce an additional block of trials to measure performance without sensory

430 stimuli after performing the task with sonification. 
433 include or exclude any number of trials, so as to refine the resolution and

434 personalization of their model. By continually using, adjusting, and decreasing the

435 threshold of error in which movements are identified as deviating from an ideal

436 performance, users might begin to optimise their movements and performance. But as

437 we observed in our study, depending on the goal of their use, certain sonification

438 strategies may affect humans differently and subsequently their movements and

439 performance.

References

Agostini, T., Righi, G., Galmonte, A., \& Bruno, P. (2004). The Relevance of Auditory Information in

Arnott, S. \& Alain, C. (2011). The auditory dorsal pathway: Orienting vision, Neuroscience \& Biobehavioural Reviews, 35 (10), 2162-2173. doi:10.1016/j.neubiorev.2011.04.005

Bieńkiewicz, M., Bourdin, C., Bringoux, C., Buloup, F., Craig, C., Prouvost, L., \& Rodger, M. (2019). The Limitations of Being a Copycat: Learning Golf Putting Through Auditory and Visual Guidance, Frontiers in Psychology 10, 92. doi:10.3389/fpsyg.2019.00092

Bonassi, G., Biggio, M., Bisio, A., Ruggeri, P., Bove, M. \& Avanzino, L. (2017). Provision of somatosensory inputs during motor imagery enhances learning-induced plasticity in human motor cortex, Scientific Reports 7, 9300.

Burchfield, R. \& S. Venkatesan (2010). A Framework for Golf Training Using Low-Cost Inertial Sensors, Proceedings of the 2010 International Conference on Body Sensor Networks. doi:10.1109/BSN.2010.46

Conan, S., Thoret, E., Aramaki, M., Derrien, O., Gondre, C., Ystad, S., \& Kronland-Martinet, R. (2014). An Intuitive Synthesizer of Continuous-Interaction Sounds: Rubbing, Scratching, and Rolling, Computer Music Journal 38, 24-37. doi:10.1162/COMJa00266

Craig, C., Delay, D., Grealy, M., \& Lee, D. (2000). Guiding the swing in golf putting, Nature, 295 -296. doi:10.1038/35012690

Dailly, Anabel, Sigrist, R., Kim, Y., Wolf, P., Erckens, H., Cerny, J., Luft, A., Gassert, R. \& Sulzer, J. (2012). Can simple error sonification in combination with music help improve accuracy in upper limb movements? Proceedings of the IEEE RAS and EMBS International Conference on Biomedical Robotics and Biomechatronics, 1423-1427. doi:10.1109/BioRob.2012.6290908.

Diedrich, F. \& J. Drischler (1957). Effect of Spanwise Variations in Gust Intensity on the Lift Due to Atmospheric Turbulenc: NACA TN 3920. 
Dubus, G. \& R. Bresin. (2014). Exploration and evaluation of a system for interactive sonification of elite rowing, Sports Engineering, 18. doi:10.1007/s12283-014-0164-0

Dyer, J., Rodger, M., \& Stapleton, P. (2016). Transposing Musical Skill: Sonification of movement as concurrent augmented feedback enhances learning in a bimanual task, Psychological Research, 81. doi:10.1007/s00426-016-0775-0

Dyer, J., Stapleton, P., \& Rodger, M. (2017). Mapping sonification for perception and action in motor skill learning, Frontiers in Neuroscience 11, 463. doi:10.3389/fnins.2017.00463

Effenberg, A., Ursula, F., Schmitz, G., Krueger, B., \& Mechling, H. (2016) Movement Sonification: Effects on Motor Learning beyond Rhythmic Adjustments, Frontiers in Neuroscience. doi:10.3389/fnins.2016.00219

Frank, C.,Land, W. M., \& Schack, T. (2013). Mental representation and learning: the influence of practice on the development of mental representation structure in complex action. Psychol. Sport Exerc. 14, 353-361. doi:10.1016/j.psychsport.2012.12.001

Gandemer, L., Parseihian, G., Kronland-Martinet, R., \& Bourdin, C. (2017). Spatial Cues Provided by Sound Improve Postural Stabilization: Evidence of a Spatial Auditory Map? Frontiers in Neuroscience 11. doi:10.3389/fnins.2017.00357.

Johnson, D., Watson, C., \& Jensen, J.. (1987). Individual differences in auditory capabilities. The Journal of the Acoustical Society of America \}, 81(2), 427-438. doi:10.1121/1.394907

Kammerer, B., Menshik, A., Erlemann, L., \& Lafortune, M. (2014). Quantifying the performance metrics of a putter, International Journal of Golf Science 4, S45-S46. (orally reported result). doi:10.1123/ijgs.2015-0007

Keogh, J. \& Hume, P. (2012). Practice conditions: How do they influence motor learning in golf? Proceedings of the 30th Annual Conference of Biomechanics in Sports, 367--370. doi:10.3389/fpsyg.2015.01981

Kleiman-Weiner, M. \& Berger, J. (2006). The sound of one arm swinging: a model for multidimensional auditory display of physical motion, Proceedings of the 12th International Conference on Auditory Display, London, UK, June 20-23, 2006.

Kohler, E., Keysers, C., Umilta, A., Fogassi, L., Gallese, V., \& Rizzolatti, G. (2002). Hearing Sounds, Understanding actions: action representation in mirror neurons, Science, 297 (5582), 846 - 848. doi:10.1126/science.1070311

Libkum, T., Otani, H., \& Steger, N. (2002). Training in timing improves accuracy in golf, Journal of General Psychology 129(1), 77-96. doi:10.1080/00221300209602034

Murgia, M., Prpic, V., O, J., McCullagh, P., Santoro, I., Galmonte, A., \& Agostini, T. (2017). Modality and Perceptual-Motor Experience Influence the Detection of Temporal Deviations in Tap Dance Sequences, Frontiers in Psychology 8: 1340. doi:10.3389/fpsyg.2017.01340

O’Brien, B., Juhas, B., Bienkiewicz, M., Pruvost, L., Buloup, F., Bringnoux, L., \& Bourdin, C. (2018). Considerations for Developing Sound in Golf Putting Experiments, Post-proceedings of CMMR 2017 Music Technology with Swing, Lecture Notes in Computer Science, Springer-Verlag Heidelberg. doi:10.1007/978-3-030-01692-0

Pataky, T. \& Lamb, P. (2018). Effects of physical randomness training on virtual and laboratory golf putting performance in novices, Journal of Sports Sciences, 36(12), 1355-1362. doi: 10.1080/02640414.2017.1378493

Richardson, A., Mitchell, A., \& Hughes, G. (2018). The effect of movement variability on putting proficiency during the golf putting stroke, International Journal of Sports Science \& Coaching. doi:10.1177/1747954118768234. 
Salako, S. E. (2006). The Declaration of Helsinki 2000: ethical principles and the dignity of difference. Medicine and Law 2, 341-354. doi:10.1515/9783110208856.233

Schaffert, N., Janzen, T., Mattes, K., \& Thaut, M. (2019) A Review on the Relationship Between Sound and Movement in Sports and Rehabilitation, Front Psycho 10: 244. doi:10.3389/fpsyg.2019.00244

Sigrist, R., Rauter, G., Riener, R., \& Wolf, P. (2013). Augmented visual, auditory, haptic, and multimodal feedback in motor learning: A review, Psychonomic Bulletin \& Review, 20 (1), 21-53. doi:10.3758/s13423-012-0333-8

Sigrist R, Fox S, Riener R, \& Wolf. P. (2016). Benefits of Crank Moment Sonification in Cycling. Procedia Engineering, 147, 513-518.

Thoret, E., Aramaki, M., Kronland-Martinet, R., Velay, J-L., \& Ystad, S. (2014). From sound to shape: auditory perception of drawing movements, Journal of experimental Psychology: Human Perception and Performance, American Psychological Association, 40(3), 983—994. doi:0.1037/a0035441

van Vugt, F. \& Tillmann, B. (2015). Auditory feedback in error-based learning of motor regularity, Brain Research 1606, 54 -67. doi: 10.1016/j.brainres.2015.02.026

Wolf, P., Sigrist, R., Rauter, G., \& Riener, R. (2011). Error sonification of a complex motor task. In $B I O$ Web of Conferences (1): 00098. EDP Sciences. doi: 10.1051/bioconf/20110100098

Wu, H., Miyamoto, Y., Castro, L., Olveczky, B., \& Smith, M. (2014) Temporal structure of motor variability is dynamically regulated and predicts motor learning ability, Nature Neuroscience 17 (2), 185-211. doi: 10.1038/nn.3616

Wulf, G. \& Shea, C. (2002) Principles derived from the study of simple skills do not generalize to complex skill learning, Psychonomic Bulletin and Review, 9(2): 185--211. doi:10.3758/BF03196276

Young, W., Rodger, M., \& Craig, C. (2014). Auditory observation of stepping actions can cue both spatial and temporal components of gait in Parkinson's disease patients, Neuropsychologia 57, 140-153. doi: 10.1016/j.neuropsychologia.2014.03.009

Zhao, H. \& Warren, W. (2014) On-line and model-based approaches to the visual control of action, Vision Research 110, 190-202.

Zwicker, E. \& Fastl, H. (1999). Psychoacoustics. Facts and Models. doi:10.1007/978-3-540-68888-4 